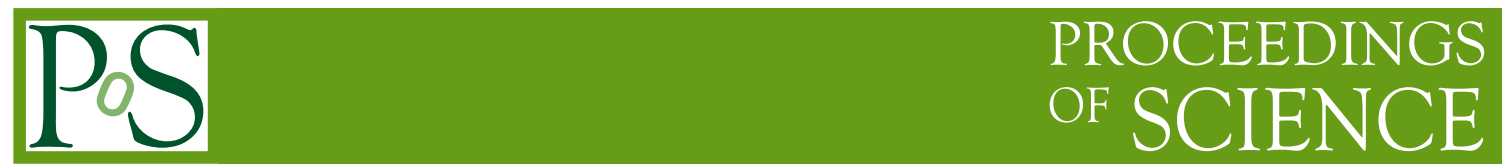

\title{
Fiber Connector for MPPC
}

Hiroyuki Kawamuko*, Tsuyoshi NAKAYA, Kazunori Nitta, and Masashi Yokoyama

Kyoto University, Japan

E-mail: kawamuko@scphys.kyoto-u.ac.jp

We report the performance of the fiber connector for the MPPC used in the T2K experiment. We develop the custom connector for a $1 \mathrm{~mm}$ diameter fiber coupled to the MPPC of the $1 \times 1 \mathrm{~mm}^{2}$ active area. With the connector, the light loss is found to be only $13 \%$. The light yield measured by the MPPC with the connector successfully satisfies our T2K requirement.

International workshop on new photon-detectors PD07

June 27-29 2007

Kobe University, Kobe, Japan

* Speaker. 


\section{Introduction to Fiber Connector}

We intensively develop Multi-Pixel Photon Counter (MPPC) for an application in the T2K neutrino oscillation experiment [1]. The T2K neutrino detector located near the neutrino beam production consists of more than 20,000 scintillation counters. The light from each scintillation counter is collected by a wave-length-shifting (WLS) fiber embedded in, and the light from the WLS fiber is transported to and measured by the MPPC. For this application, we develop a custom light-connector of the WLS fiber coupled to the MPPC. A schematic view of the scintillator with the WLS fiber, the connectors, and the MPPC is shown in Figure 1. In T2K, we use the $1 \mathrm{~mm}$ diameter fiber and the MPPC with the $1 \times 1 \mathrm{~mm}^{2}$ active area ${ }^{1}$. Since the area of the MPPC is so close to the area of the fiber, we must align the fiber well matching to the MPPC. The custom light connector of the fiber to the MPPC is designed for this purpose. As shown in Figure 1, the fiber connectors consist of two parts: one part holds the MPPC at the bottom of the funnel, and the other part bonds the fiber. The two parts can be attached without glue nor screw. So the connection of two parts (the fiber and the MPPC) is simple, well-aligned, and robust.

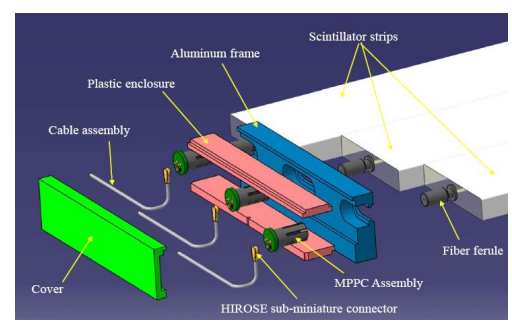

Figure 1: A schematic view of the scintillator in T2K with the WLS fiber, the connector, and the MPPC.

\section{Measurement of light loss with the connector}

We expect the light loss with the connectors since the surface of the fiber is not perfectly matched to the surface of the MPPC. Especially, the loss could be caused by a few hundred micron air gap between the fiber and the MPPC due to the position of the MPPC device in the package. We measured the light loss with the connectors in the test setup as shown in Figure 2. The WLS fiber of $1 \mathrm{~mm}$ diameter was illuminated by LED. The light output from the fiber was measured by the MPPC with and without the connector. The ratio of the light output with and without the connector was measured to be 0.87 , which results in $13 \%$ light loss with the connector. The $13 \%$ light loss could be explained by the loss due to the air gap between the fiber and the MPPC. We measured the light loss by varying the bias voltage applied to the MPPC. No significant variation and good reproducibility were found.

\section{Measurement of Photon-Detection-Efficiency (PDE) with the connector}

We are much interested in the net light output from the scintillation counter, and the performance of the MPPC relative to that of a PMT. With our experience of the K2K SciBar detector [2],

\footnotetext{
${ }^{1}$ Later, we decide to use the MPPC with the $1.3 \times 1.3 \mathrm{~mm}^{2}$ active area.
} 

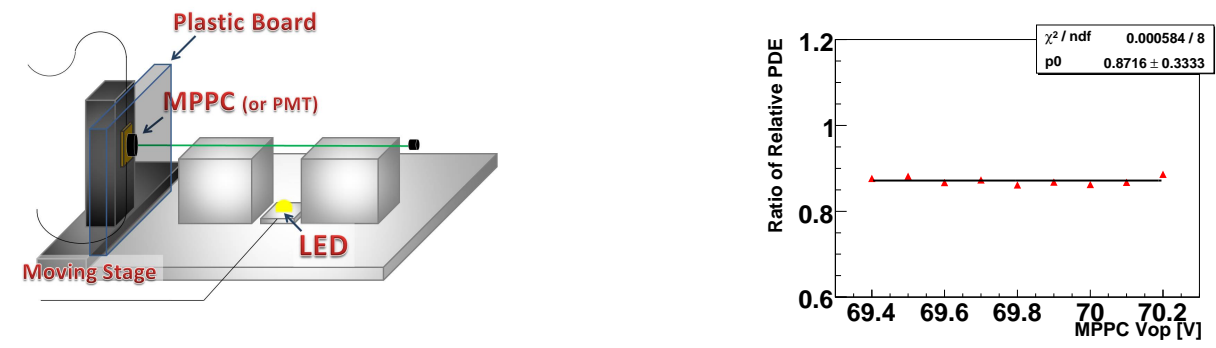

Figure 2: Experimental setup to measure the effi-

ciency of the fiber connector to the MPPC. At the end Figure 3: The ratio of the light outputs from the WLS of the WLS fiber illuminated by LED, the MPPC's fiber by using MPPC's with and without the connecwith and without connector were attached. For the tors as a function of the bias voltage applied to the calibration purpose, we also used a PMT. MPPC.

we require the MPPC to have the equal or better light yield than a PMT in T2K. We measured the light output from the $1 \mathrm{~mm}$ diameter WLS fiber by the MPPC relative to a PMT. The experimental setup is shown in Figure 4. The WLS fiber was illuminated by LED at the center, and the MPPC with the connector and a PMT were connected at both sides of the fiber. We measured the twice more light yield by the MPPC than by the PMT as shown in Figure 5. The photon-detectionefficiency (PDE) of the MPPC with the connector is found to be twice as high as the quantumefficiency (QE) of the PMT for green light from the WLS fiber. We repeated the measurement for 15 hours, and we had the stable results.
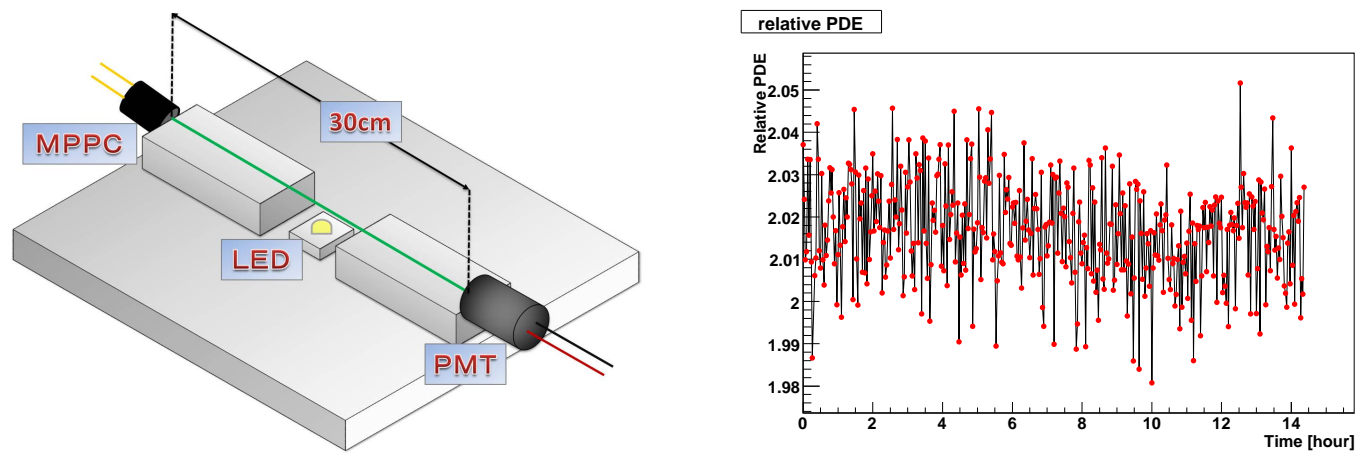

Figure 4: Experimental setup to measure the light Figure 5: Relative Light yield measured by the MPPC output by the MPPC to the PMT

The light yield by the MPPC with the connectors is satisfied to the T2K requirement. Since this measurement includes the effect of the light loss reported at Section 2, we conclude that the design of the connector is also satisfactory for $\mathrm{T} 2 \mathrm{~K}$ with the $13 \%$ light loss .

\section{References}

[1] S. Gomi et al., "Research and development of MPPC for the T2K experiment", contribution in these proceedings.

[2] K. Nitta et al., Nucl. Instrum. Meth. A 535 147-151 (2004). 